\title{
Parametric Study of Spherical Micro-lens Array
}

\author{
R.F.Shyu, a , C. T. Pan ${ }^{2, b}$, S. C. Lin $^{2, c}$ \\ ${ }^{1}$ Department of Mechanical Manufacturing Engineering, National Formosa University \\ 64 Wen-Hua Road Huwei, Yunlin, Taiwan \\ ${ }^{2}$ Department of Mechanical and Electro-Mechanical Engineering, Center for Nanoscience \& \\ Nanotechnology, National Sun Yat-Sen University, Kaoshiung 804, Taiwan
}

arfshyu@nfu.edu.tw, bpanct@mail.nsysu.edu.tw, 'cm933020040@student.nsysu.edu.tw

Keywords: micro-lens, extra-hardness, Ni-Co alloy electroplating, optical measurement.

\begin{abstract}
This study presents a concept to fabricate micro-lens devices using high-aspect-ratio lithography, extra-hardness electroplating, and hot embossing processes. A bath of electroplating electrolyte will be formulated to fabricate micro-optics mold inserts with extra-hardness Ni-Co alloy. It is a novel method to increase the life of the mold insert during fabricating micro-lens devices. With this high hardness, the mold inserts can resist high abrasiveness and wearness so as to extend the mold cycle life and reduce the idle time of replacing mold plates during fabrications. Therefore, the process of fabrications of micro-lens can be more cost-effective. In this study, parametric effects of reflow time, and temperature on micro-lens profiles will be characterized and discussed. Finally, the optical properties such as focal length of developed micro-lens will be measured and tested.
\end{abstract}

\section{Introduction}

Integrated micro-lens array is interesting for various applications, in the field of optical communication, optical storage and digital displays. Micro-lens plays a very important part in micro optical component such as video cameras, video phones, compact-disk data storage, robotic vision, optical scanner, and high-definition projection displays. The major objective of micro-lens is to increase the coupling efficiency of optical system, such as enhancing the brightness of backlight module (BLM) for liquid crystal display (LCD) and increasing the efficiency of optical switches. Micro-lens technology is used to raise $25 \%$ light output in a laptop display [1]. The fabricative method of high aspect ratio micro-lens is required to fit the rapid growth of commercial devices.

In recent years, interest has grown in the study of micro-lens arrays for a wide variety of applications. These diverse applications promote many new methods discussed by researchers related to fabrication of a micro-lens array. The established methods of refractive micro-lens fabricated are photoresist reflow technique $[2,3,4,5]$. Firstly, photoresist cylinders were fabricated by lithography process, and then those were heated over the glass temperature (Tg) of the photoresist. The photoresist changed from the cylinders to half-spherical shapes ideally by surface tension, and the half-spherical micro-lens array was obtained. Yang et al. used the modified reflow process to fabricate micro-lens with high fill-factor, and the gapless square micro-lens array was obtained on the silicon substrate [6]. The special shape such as gapless hexagonal micro-lens array was fabricated by thermal reflow process and the mathematical formula was computed [7]. Next process of molding is required to fabricate the micro-optical component in mass production [8,9]. On the other hand, Microjet system technology offers by printing the number of droplets on the substrate and forming hemisphere micro-lens array by surface tension $[10,11,12,13,14]$. This method is simple but the unity is not uniform, its unity dependent on the apparatus precision. Furthermore, fabrication of micro-lens by gray level mask were described [15], gray level mask can fabricate all shape micro-lens by design the mask pattern. However, it must need to consider the exposure dose of a photoresist to be designed, and it is too difficult. 
This study presents a new process to design and fabricate high aspect ratio micro-lens array. The process have the advantage of cheapness, uniformity and easy to design the mask. The process mainly includes a high-aspect-ratio UV lithography, a photoresist reflow technique, Ni-Co electroplating with extra-high hardness, hot embossing process as well as optical measurement. In order to understand micro-lens forming mechanism, we set different photoresit (AZ4620) reflow temperature and period, and also control electroforming condition. To discuss above described parameters that will affect the micro-lens forming mechanism. In addition, Ni-Co electroplating process with hardness larger than Hardness of Vicker (Hv) 560 is developed. With this mold for hot embossing process, micro-lens array can be obtained.

\section{Process}

When the temperature is above the glass transition temperature (Tg), photoresist molecule is movable. Baking temperature above Tg will provide thermal energy to make photoresist molecule do thermal motion. The thermal motion will enable the undue exposure molecule to rearrange. All photoresist columns will form hemispherical micro-lens due to the surface tension effect.

In the recent years, the development of thick film photoresist material and novel method of photoresist spin coating improve the precision of UV thick film fabrication technique. Thick film photoresist acts as the mold inset of electroforming in LIGA-like process and the thick film photoresist is known as primary master mold. The inverse mold of primary master mold which is known as secondary master mold to emboss microstructure. In order to apply in electroforming process, we need to consider thick film photoresist that can resist erosion by electroforming solution and the thickness of photoresist is higher than the thickness of the electroforming microstructure to maintain the roughness.

\section{Process Procedures}

The exposure process of thick film. Firstly, 4 inch silicon wafer as substrate was prepared including RCA process and dehydration baking etc. Then, the wafer was sent to prime the HDMS and spin coating photoresist AZ4620. The spin rate was $1000 \mathrm{rpm}$ and the thickness of the photoresist was 22 $\mu \mathrm{m}$ in 30 seconds. Silicon subtract was sent to the mask aligner to exposure about at 36 seconds after soft-baking at $90{ }^{\circ} \mathrm{C}$. The dosage of the exposure was $360 \mathrm{~mJ}$. After developing, the column array on the silicon substrate could be defined.

Reflow technique. Finally, this structure was heated to a temperature above the photoresist glass temperature. The photoresist columns were melted and the surface tension effect changed the photoresist profile into a spherical shape as so-called reflow process. The original column array was changed into the half-spherical micro-lens array. In this experiment, we consider two important parameters. The first step, we set the same heating period and change the heating temperature to discuss the reflow effect. The second step, we set the same heating temperature and change the heating period, and discuss two set results by period and temperature to get optimal parameter to improve the stability and precision of the reflow process.

Electroforming. The hardness of the Ni-Co mold over Hv 560 can be obtained and its residual stress after electroplating process was below $1.5 \mathrm{~kg} / \mathrm{mm}^{2}$. Then $\mathrm{Ni}$ thin film was sputtered on the resist template surface served as seed layer. Then $\mathrm{Ni}-\mathrm{Co}$ electroplating technique was used to form the Ni-Co mold with $1 \mathrm{~mm}$ in thickness, followed by chemical mechanic polishing (CMP) process to reduce the total thickness variation of Ni-Co mold after electroplating process. The hardness of the Ni-Co mold over Hv 560 can be obtained and its residual stress after electroplating process was below $1.5 \mathrm{~kg} / \mathrm{mm}^{2}$.

Hot embossing. In the study, experiment parameters include hot embossing time, applied loading force, and hot embossing temperature. The PMMA sheet with $1 \mathrm{~mm}$ in thickness is purchased from Hsintou Company in Taiwan. Its glass transition temperature and average molecular weight are 
$105^{\circ} \mathrm{C}$ and 600,000 [g/mol], respectively. In the experiment, PMMA is used for hot embossing process. The reason to select PMMA as micro-lens material is that the material is very suitable for optical devices because of physical properties, optical properties and ability for IR coating.

\section{Results and discussion}

The effect of various temperatures with constant period. We maintain the same heating period and change the heating temperature. In this experiment, we heat photoresist column for 6 hours at various temperature. The relation between heating temperature and the diameter of reflowed micro-lens is shown in figure 1 . Besides, we also considered the focal length by various reflowed temperature as shown in figure 2 .

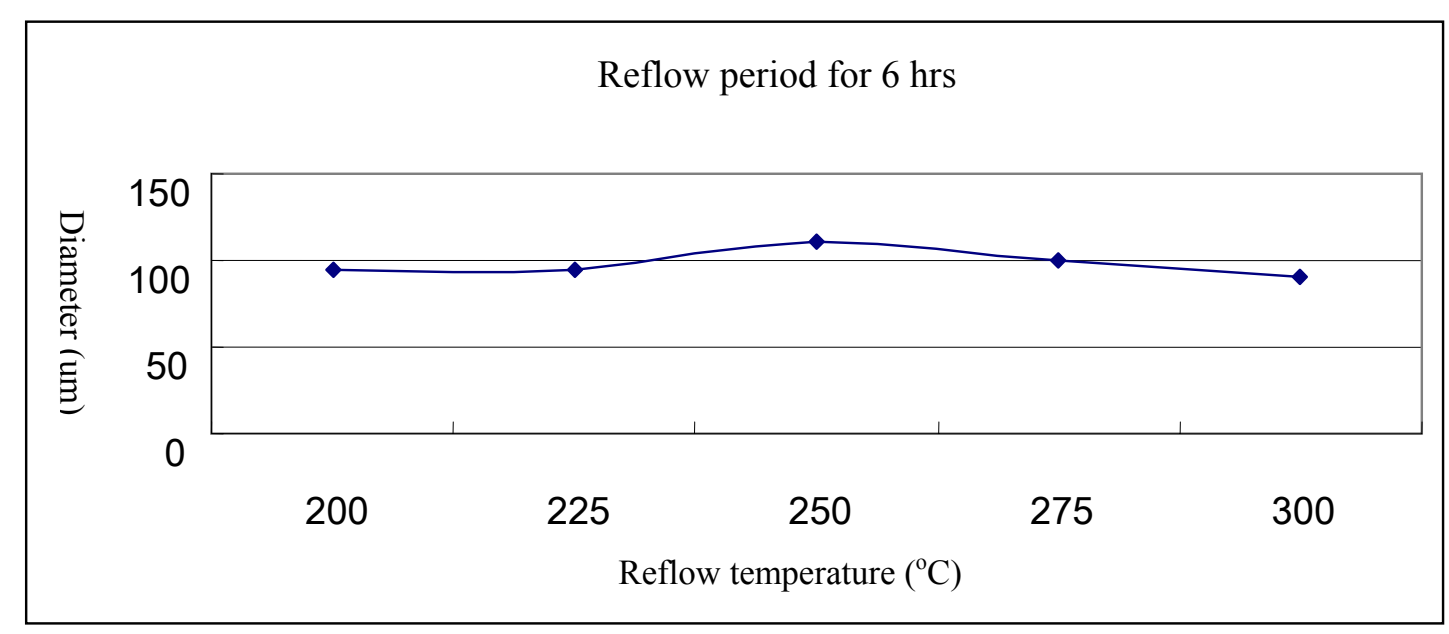

Fig. 1. Reflow period for $6 \mathrm{hr}$, Micro-lens diameter as function of reflow temperature.

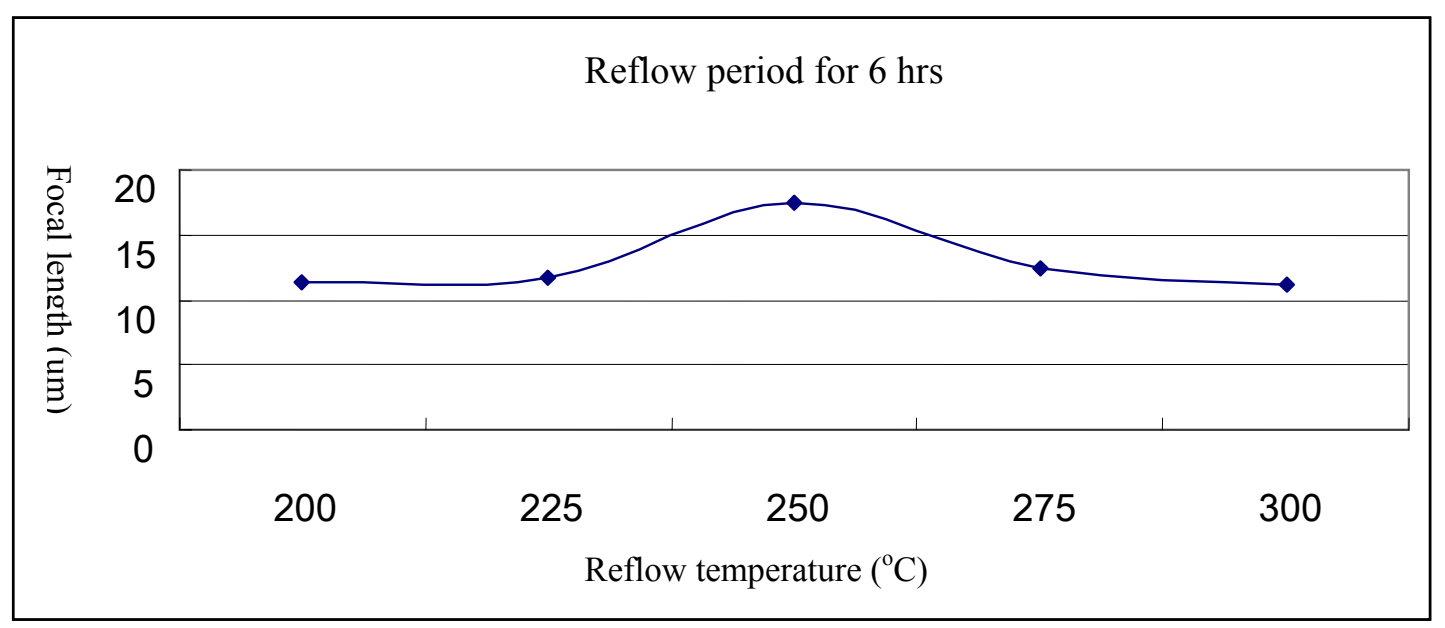

Fig. 2. Reflow period for $6 \mathrm{hr}$, Focal length as function of reflow temperature.

As shown in figure 1, the diameter of the micro-lens will increase as the heating temperature increases. When the heating temperature is above the threshold value, the diameter of micro-lens will decrease. AZ4620 will show colloid property when the temperature above Tg. As temperature increases, the micro-lens shape becomes smooth and liquid-like status due to the surface tension effect. When the temperature increases further to the threshold value, the photoresist will solidify and the photoresist shape will shrink due to the evaporation of the photoresist.

The changed curve of diameter of the micro-lens is similar to the curve of focal length. As temperature changed the diameter of micro-lens changed due to surface tension effect, and the micro-lens shape will affect the focal effect of the micro-lens. 
The effect of various reflow period with constant temperature. We maintain the same reflowed temperature and change the heating period. In this experiment, we set the reflowed temperature at $225^{\circ} \mathrm{C}$ and various heat period .The relation between heating period and the diameter of reflowed micro-lens is shown in figure 3 . Besides, we also considered the focal length by various reflowed periods as shown in figure 4.

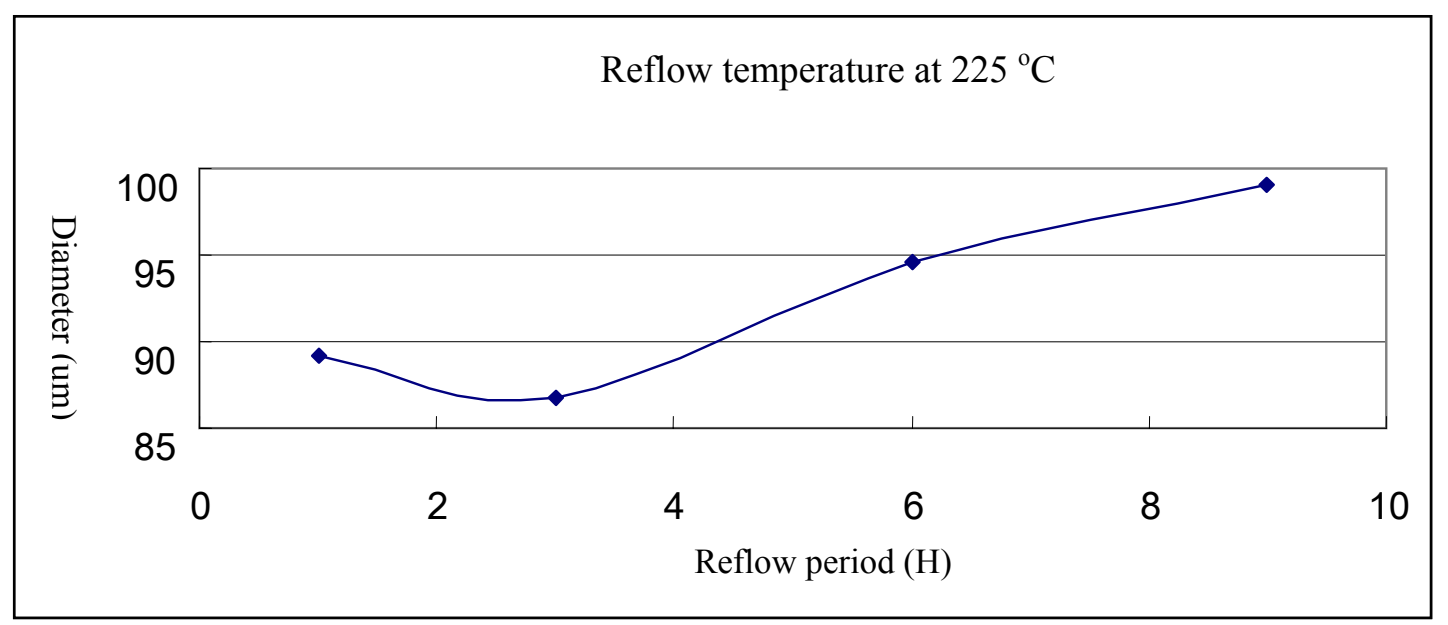

Fig. 3. Reflow temperature set at $225^{\circ} \mathrm{C}$, Micro-lens diameter as function of reflow period.

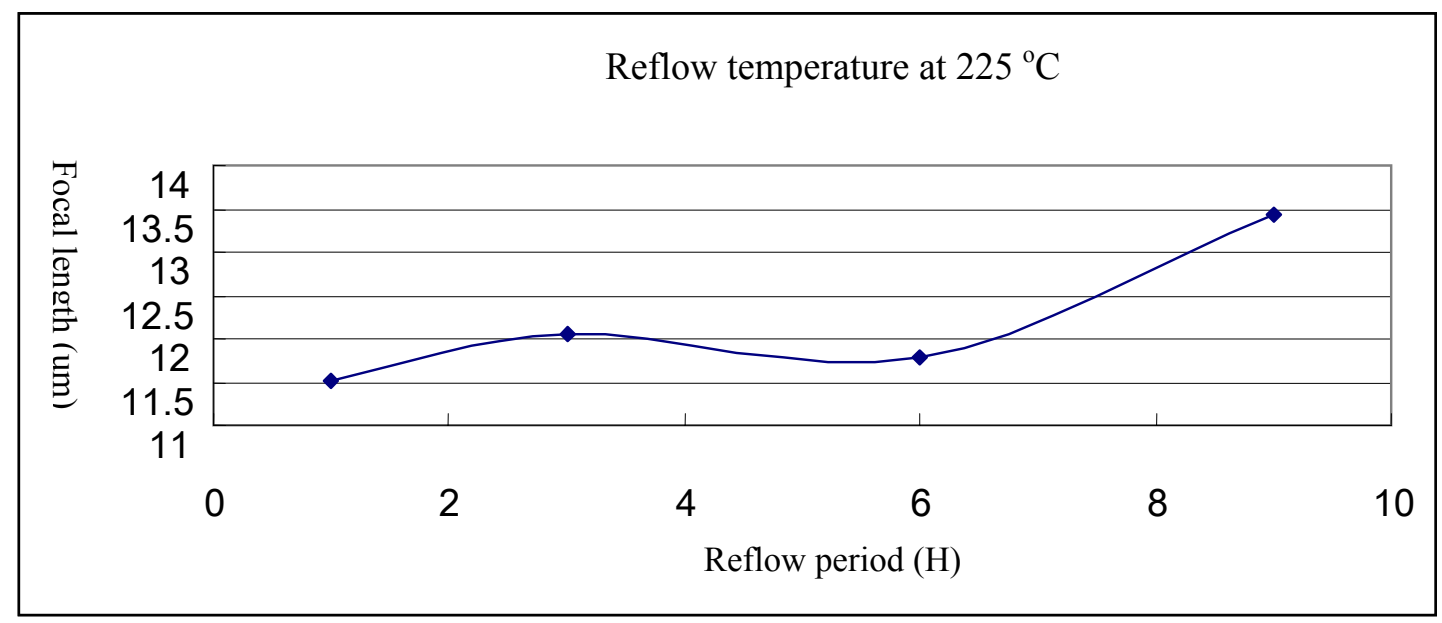

Fig. 4 . Reflow temperature set at $225^{\circ} \mathrm{C}$, Focal length as a function of reflow period.

The result shows that the heating period results in an increase in the micro-lens focal length. AZ4620 shows colloid profile when the temperature above its Tg. As temperature increases the micro-lens shape become smooth due to the surface tension effect.

In this experiment, we also can obtain that the changed curve of diameter of the micro-lens is similar to the curve of focal length. As temperature changed the diameter of micro-lens changed due to surface tension effect, and the micro-lens shape will affect the focal effect of the micro-lens.

The reflowed micro-lens array is shown as Figure 5, and we can fabricate the electroforming Ni-Co mold shown as Figure 6. After hot embossing process, the PMMA optical thin film of high-aspect-ratio micro-lens array was obtained shown as Figure 7. 


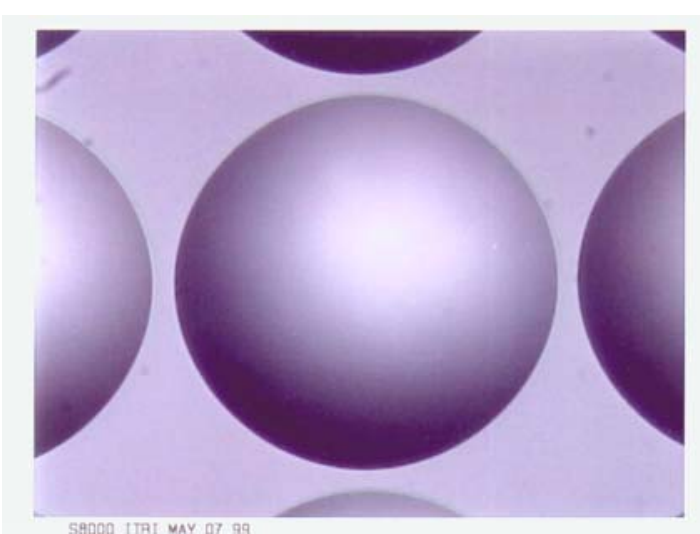

Fig. 5. The reflowed micro-lens array.

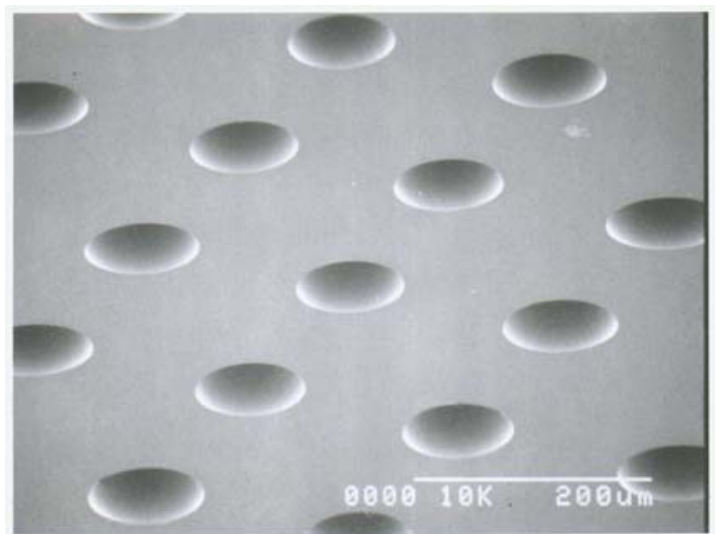

Fig. 6. Electroforming Ni-Co mold.

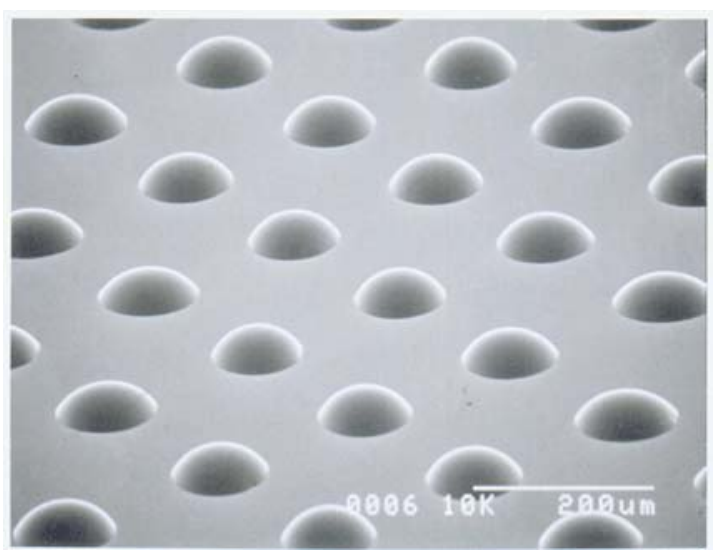

Fig. 7. Hot embossing micro-lens array.

\section{Conclusions}

The parameters of reflowed process were studied in this paper. The micro-lens shape does not show obvious change under controlled high temperature span. On the other hand, the reflow mechanism can not happen if the heating temperature is too low. The reflow process affects the photoresist column shape, thus we discuss the reflowed diameter under various heating temperature and reflow period .In this study, the micro-lens array with high fill-factor have been fabricated, it can improve the brightness and diffuseness. And the hardness of the Ni-Co mold over Hv 560 can be obtained and its residual stress after electroplating process was below $1.5 \mathrm{~kg} / \mathrm{mm}^{2}$. The resist template could be transferred into Ni-Co mold for subsequent mass production. 


\section{Acknowledgments}

The authors would like to thank Dr. Tung-Chuan Wu at MIRL of ITRI in Taiwan for his guidance, and National Science Council (NSC) for their financial supports to the project (granted number: NSC93-2622-E-110-003-CC3, NSC93-2212-E-110-028 and NSC93-2212-E-110-029).

\section{References}

[1] Ezell, B., Information Display. A5, 42 (2001).

[2] Popovic, Z. D., Sprague, R. A. and Connell, G. A. N., Applied Optics. A27, 1281 (1988).

[3] Hutley, M. C., Journal of Modern Optics. A37, 253 (1990).

[4] Choi, J. O., Morse, J. A., Corelli, J. C., Silverman J. P. and Bakhru H., J. Vac. Sc. Technol. A6, 3633 (1988).

[5] Borrelli, N. F., Morse, D. L., Bellman R. H. and Morgon W. L., Applied Optics. A24, 2520 (1985).

[6] Yang, H., Chao, C. K., Wei, M. K., Lin, C. P., Journal of Micromechanics and Microengineering. A14, 1197 (2004).

[7] Lin, C. P., Yang, H., Chao, C. K., Journal of Micromechanics and Microengineering. A13, 775 (2003).

[8] Yang, H., Chou, M. C., Yang, A., Mu, C. K. and Shyu, R. F., Proc. of SPIE. A3739, 178 (1999).

[9] Yang, H., Pan, C. T. and Chou, M. C., Journal of Micromechanics and Microengineering. A11, 94 (2001).

[10] Lee, S. K., Lee, K. C. and Lee, S. S., Journal of Micromechanics and Microengineeing. A12, 334 (2002).

[11] Cox, W.R., Chen, T., Ussery, D., Hayes, D.J., Tatum, J.A. and MacFarlane, D.L., Optics Communications. A123, 492 (1996).

[12] Cox, W.R., Chen, T. and Hayes, D.J., OSA Optics \& Photonics News. A12(6), 32 (2001).

[13] Cox, W.R., Chen, T. and Grove, M.E., Proceedings of SPIE. A4292, 208 (2001).

[14] Cox, W.R., Chen, T., Ussery, D. W., Hayes, D. J. and Hoenigman, R. F., SPIE Proceedings. A2687, 89 (1996).

[15] Cox, W.R., Hayes, D. J., Chen, T., Trost, H. J., Grove, M.E., Hoenigman, R.F. and MacFarlane, D.L., IMAPS International Journal of Microcircuits \& Electronic Packaging, A20(2), 89 (1997).

[16] Oppliger, Y. et al., Microelectronic Engineering. A23, 449 (1994). 